\title{
Minimizing the Total Cost of WAMS Using Artificial Electric Field Algorithm
}

\author{
Batool. B. Al-Khraisat ${ }^{1}$, Ali. S. AL-Dmour ${ }^{2 *}$, Khaled. S. Al Maitah ${ }^{3}$ \\ Electrical Engineering Department, College of Engineering, Mutah University, Karak, Jordan \\ Received: January 15, 2021. Revised: April 7, 2021. Accepted: April 12, 2021. Published: April 19, 2021.
}

\begin{abstract}
Wide area monitoring system (WAMS) is one of the most important technologies in modern power systems, it gathers the synchronized time stamped measurements from wide area across power system through a high reporting measuring device such as synchronized phasor measurement units (PMUs).Due to the high cost of PMU itself and its installation cost, it is often difficult to install PMU at each bus in the power system. However, there are several approaches to solve the optimal PMU placement (OPP) with various optimization algorithms. In this paper, a realistic cost model has been proposed to provide optimal PMUs placement (OPP). The proposed model has been solved using artificial electric field algorithm (AEFA), which is a novel optimization algorithm, it was proposed by 2019. In this paper, a binary form of AEFA has been proposed to solve the proposed cost model. AEFA has been implemented in MATALB programming environment, and the results demonstrate that the proposed cost model provides a realistic view of WAMS cost. Additionally, the results have been compared with other optimization algorithm, and it demonstrates that the AEFA is efficient to solve the OPP problem.
\end{abstract}

Keywords - optimal PMUs placement; wide area monitoring system; artificial electric field algorithm.

\section{INTRODUCTION}

Due to the rapid development of power grid, it essential to be smarter rather than before. The transformation from bulk grid to smart grid need to integrate the information and communication layer to the existence conventional power layer. This transformation allowing an accurate and real time monitoring and controlling. Wide Area Monitoring System (WAMS) is one of the most important technologies in modern power systems, and it considered as a one of the primary systems to develop the existence power grid to be smart grid. WAMS capable to provide a real-time view of the dynamic behavior of the electrical variable. It gathers the synchronized time stamped measurements from wide area across power system through a high reporting measuring device such as synchronized phasor measurement unit (PMU). When a PMU has installed at a bus, it can measure the phase voltage of that bus and the phase current of all branches connected to that bus.

Due to the high cost of PMU itself and its installation, it is often difficult to install PMU at each bus in the system. Additionally, the determination process of the required number PMUs and the most appropriate placement of these units need extended deeply study[1]. From this point, many researchers 
have tackled the question of optimal PMUs placement (OPP) with different approaches[2,3]. The main objective of the optimal PMUs placement problem is determining the minimum number of PMUs at the system buses to provide full observability. It should be noted that, the full observability means that all buses voltages, branches currents, and phasor angles are measured[4].

In general, there are two main separated scope for OPP problem available in the literature, the first one deals with the OPP problem formulation and the second scope deals with the optimization techniques that have been used to solve the OPP problem.

In reference [5], the authors used number of PMUs as an objective function to solve the OPP problem in many IEEE test systems. OPP problem with additional contingency constraint was proposed in [6], the authors considered single PMU outage and single line outage as additional constraint in their optimization formulation. Although the OPP problem were solved by many researchers through minimized the number of PMUs, other researcher used the PMU installation cost as an objective function. For example, the authors in [7] assumed that the cost of PMU installation is the PMU cost only. Practically, there is no different between PMU cost minimization and PMU number minimization. On other hand, other researchers assumed that the total cost of PMU depends not only on the PMU cost but also depends on communication network. Consequently, the authors in [8] proposed a cost model to solve the OPP problem based on PMU cost and communication infrastructure, their model based on minimized the distance between PMUs and phasor data concentrator(PDC).

Additionally, other cost factors have been considered in OPP problem formulation such as PMU channel capacity, and substation preparation in [9]. The authors in [9] assumed the practical implementation WAMS may need to upgrade some of substation auxiliary component such as installing new current transformer (CT) and voltage transformer (VT), upgrade the communication infrastructure in some substation, and upgrade security system. In this case the substations which have sufficient readiness must have more chance to install PMU. The authors in [10] have suggest OPP problem formulation by minimize the total cost with considering the communication infrastructure, security upgrade and measurement transformer cost (VT and CT).

As stated early, the second scope of OPP problems in the literature centered about the optimization technique that are used to solve the OPP problem. The OPP problem is an optimization problem, which is consists of objective function and set of constraint. Thus, an optimization technique must be used to solve the OPP problem. Therefore, many of optimization techniques are used to solve the OPP problem. For example, the work presented in [6] used an integer linear programming (ILP) to solve the OPP problem, the authors formulated the OPP as linear optimization problem. The optimal PMU placement with other contingency is modeled as linear formulation in [11]. The authors in[12]considered a conventional power flow and injection measurements to solve OPP problem using (ILP). A binary integer linear programming (BILP) model for simultaneous placement of PMUs and traditional power flow measurements is formulated in [13]. A multi-objective integer quadratic programming (IQP) formulation of the OPP problem that minimizes the number of PMUs with observability constraint and maximizes the measurement redundancy is presented in [14].

Multi-stage simulated annealing (SA) for OPP problem was proposed in [15], where the authors proposed a multistage SA and compares the proposed one with conventional SA. Additionally, genetic algorithm (GA) is used to solve the OPP problem with various operation condition of smart grid in [16] and[17]. GA was used with fault tolerance approach to solve the OPP problem in paper[18]. Particles swarm optimization (PSO) was used by authors reference[19] to solve the OPP problem. Multiple solutions of OPP were used to select the best one using PSO in [3], where authors considered power system aspects to select the best solution from local solutions. Cuckoo optimization algorithm (COA)was used to solve the OPP problem in [20], where authors proposed a binary structure of COA to solve the OPP problem, and single line outage are considered in them study.

In this paper, a realistic cost model has been proposed. The proposed model includes the PMU cost, PDC cost, and additional cost, where the 
additional cost in the proposed model represents the needed to upgrade some devices such as VT, CT, communication devices needed to upgrade the communication infrastructure in the substations. The proposed model has been solved using modified artificial electric field algorithm (AEFA), where based in authors knowledge this algorithm did not used yet to solve any type of OPPs problem. AEFA is a novel which is a novel optimization algorithm, it was proposed in[21].

The organization of the rest of this paper as follows. Section 2 reviewed the AEFA and presents the flow chart of original AEFA. Section 3 presents the modified AEFA to solve the proposed OPP model, where the proposed cost model is presented in section 4. The results and discussion have been presented in section 5, and finally the paper conclusion has been presented in section 6 .

\section{ARTIFICIAL ELECTRIC FIELD ALGORITHM}

Heuristics and metaheuristics are two main classes of the optimization techniques available in the literature. Metaheuristics optimization techniques are more popular in recent year, that because it easy to implement and have high computation power. The metaheuristics algorithms are inspired from natural phenomena.

As any metaheuristics optimization techniques, AEFA is inspired from electrostatic force between charge particles [21]. Coulomb' law of electrostatic force states that an electrostatic force (attraction or repulsion) between two particles of charge is directly proportional to the product of their charges and inversely proportional to the square of the distance between their positions[22]. However, Coulomb's law of the electrostatic force between two objects of charges $Q_{i}$ and $Q_{j}$ is given by[22],

$$
F_{i j}=K \frac{Q_{i} Q_{j}}{R^{2}}
$$

where $F_{i j}$ is the magnitude of electrostatic force, $K$ is the Coulomb's constant, $Q_{i}$ and $Q_{j}$ are charges of $i^{\text {th }}$ and $j^{\text {th }}$ objects, respectively. $R$ is the distance between two charges $Q_{i}$ and $Q_{j}$. Then the electric field around charge $Q_{i}$ is given by:

$$
E_{i}=\frac{F_{i j}}{Q_{i}}
$$

Based on Newton's second law of motion, when force $F_{i j}$ is applied to an object of mass M, its velocity will vary. The variation of velocity or acceleration $a_{i}$ of any charge $i$ is equal to the force exerted upon the system divided by the mass of the particle $\mathrm{M}$ as:

$$
a_{i}=\frac{F_{i j}}{M}
$$

Where, $F_{i j}$ is the magnitude of electrostatic force and $M$ is the mass of the particle $i$. To understand the AEFA, Let the position of $i^{\text {th }}$ particle in the $d$ dimensional search space is $X_{i}=\left(x_{i}^{l}, x_{i}{ }_{i}, \ldots . ., x_{i}^{d}\right)$ for $i=1,2,3, \ldots . ., N$, where $x^{d}{ }_{i}$ is the position of $i^{\text {th }}$ particle in the $d^{\text {th }}$ dimension. AEFA uses the position of the global best fitness obtained by all the charged particles as well as the personal best fitness history of each particle. The position of the best fitness value obtained by any particle $i$ at any time $t$ is given by:

$p_{i}^{d}(t+1)=$

$\left\{\begin{array}{l}p_{i}^{d}(t) \text { if } f\left(p_{i}^{d}(t)\right)<f\left(x_{i}^{d}(t+1)\right) \\ x_{i}^{d}(t+1) \text { if } f\left(x_{i}^{d}(t+1)\right)<f\left(p_{i}^{d}(t)\right)\end{array}\right.$

Now, by reformulate the Coulomb's law, the force acting on the charge $i$ from charge $j$ is defined as in Eq. (5).

$$
F_{i j}^{d}(t)=K(t) . \frac{Q_{i}(t) Q_{j}(t)\left(p_{j}^{d}(t)-x_{i}^{d}(t)\right)}{R_{i j}(t)+\epsilon}
$$

Where, $Q_{\mathrm{i}}(\mathrm{t})$ is the charge of $i^{\text {th }}$ particle and $Q_{j}(\mathrm{t})$ is the charge of $j^{\text {th }}$ particle, $K(t)$ is the Coulomb's constant, which is function of time $t, R_{i j}(t)$ is the Euclidian distance between particles $i$ and $j$ particles.

The Euclidian distance is the length of a line segment between the two points in space. It can be calculated from the Cartesian coordinates of the points using the Pythagorean theorem. However, the Euclidian distance between particles $i$ and $j$ particles can be calculated as, 


$$
\begin{aligned}
R_{i j}(t)=\left\|x_{i}(t), x_{j}(t)\right\|_{2} \\
=\sqrt{\sum_{k=1}^{N}\left|x_{i}^{k}(t)-x_{j}^{k}(t)\right|^{2}}
\end{aligned}
$$

Where, $x_{i}(t)$ and $x_{j}(t)$ are the positions of $i$ and $j$ particle, respectively is the number of elements in each particle. The Coulomb's constant $K(t)$ in Eq. (5) is a function of iteration and maximum iteration, and it given by:

$$
K(t)=K_{o} e^{-\alpha\left(\frac{\text { iter }}{\text { maxiter }}\right)}
$$

Where, $K_{o}$ is the initial value of Coulomb's constant, iter is the current iteration, maxiter is the maximum number of iterations, and $\alpha$ is learning rate of the algorithm. The values $K_{o}$ and $\alpha$ represent setting parameters, any change in this parameter will effect on the algorithm speed and it results. However, it is recommended to choose the Coulomb's constant $K_{o}$ as a high value, it is decreasing iteration by iteration[21].The total electric force acts on the $i^{\text {th }}$ particle by all the other particles at any time $t$ in a d-dimensional given by:

$$
F_{i}^{d}(t)=\sum_{j=1, i \neq j}^{N} \operatorname{rand}() F_{i j}^{d}(t)
$$

Where $\operatorname{rand}()$ is a random number in the interval $[0,1], N$ is the number of particles in the search space, and $F_{i j}^{d}(t)$ is given by Eq. (5). The electric field of the $i^{\text {th }}$ particle at any time $t$ and in $d^{\text {th }}$ dimension is given by Eq. (9).

$$
E_{i}^{d}(t)=\frac{F_{i}^{d}(t)}{Q_{i}(t)}
$$

Where, $Q_{i}(t)$ is the charge of $i^{t h}$ particle and $F_{i}^{d}(t)$ is the total electric force acts on the $i^{\text {th }}$ particle by all the other particles, which is given by Eq. (8). The acceleration $a_{i}^{d}(t)$ of any charge $i$ at any time $t$ and in $d^{\text {th }}$ dimension equal to the force exerted upon The flow chart of AEFA is presented in Fig.1. the system divided by the mass of the particle $M_{i}(t)$ as:

$$
a_{i}^{d}(t)=\frac{Q_{i}(t) E_{i}^{d}(t)}{M_{i}(t)}
$$

Where, $Q_{i}(t)$ is the charge of $i^{t h}$ particle, $E_{i}^{d}(t)$ is the electric field of the $i^{t h}$ particle at any time $t$ and in $d^{t h}$ dimension, and $M_{i}(t)$ is the unit mass of $i^{t h}$ particle at any time $t$. Thus, the velocity $V_{i}^{d}$ and position $X_{i}^{d}$ of the particles are respectively updated as:

$$
\begin{gathered}
V_{i}^{d}(t+1)=\operatorname{rand}() V_{i}^{d}(t)+a_{i}^{d}(t) \\
X_{i}^{d}(t+1)=X_{i}^{d}(t)+V_{i}^{d}(t+1)
\end{gathered}
$$

The charge of each particle $Q_{i}(t)$ is calculated by evaluate the fitness function and assuming that charge of each particle is equal. The charge $Q_{i}(t)$ at any time $t$ can be calculated as:

$$
Q_{i}(t)=\frac{q_{i}(t)}{\sum_{i=1}^{N} q_{i}(t)}
$$

Where $q_{i}(t)$ is given by:

$$
q_{i}(t)=\exp \left[\frac{\left(f i t_{p i}(t)-\operatorname{worst}(t)\right)}{(\operatorname{best}(t)-\operatorname{worst}(t))}\right]
$$

where $f i t_{i}(t)$ is the fitness value of $i^{\text {th }}$ particle at any time $t$. best $(t)$ and $\operatorname{worst}(t)$ for minimization problem are defined respectively as:

$$
\operatorname{best}(t)=\min \left(f i t_{j}(t)\right), \text { where } j \in(1,2, \ldots . . N)
$$

$\operatorname{worst}(t)=\max \left(f i t_{j}(t)\right)$, where $j \in(1,2, \ldots \ldots . N)$ 


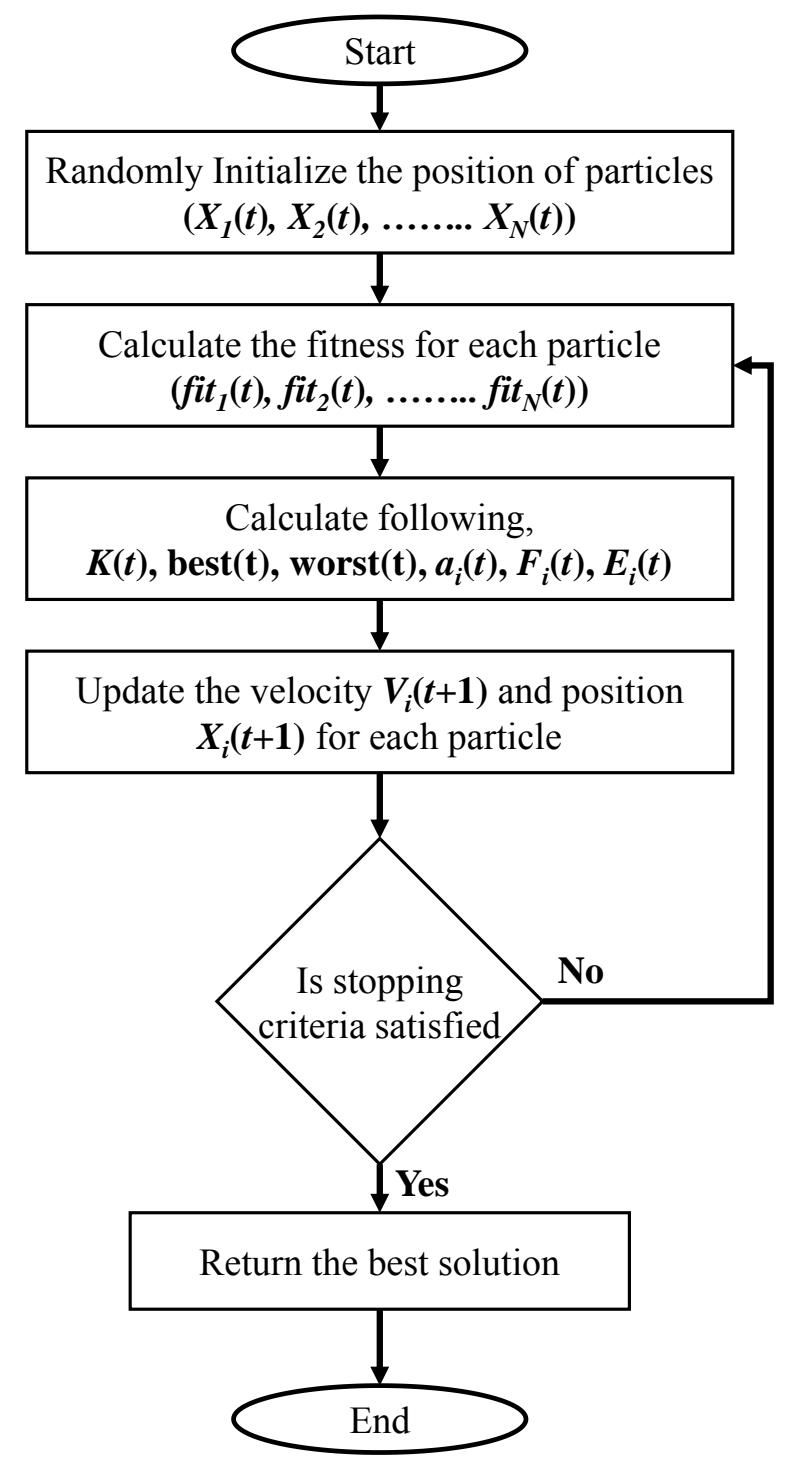

Fig. 1. Flow chart of AEFA

\section{MODIFIED AEFA TO SOLVE OPP PROBLEM}

To solve the OPP problem using AEFA, a modification has been applied to the existence AEFA. Where the existence AEFA is constructed to solve continuous optimization problems, whereas the OPP problem is a discrete optimization problem. The term of continuous or discrete optimization refers to the expected values of the optimized variable. Additionally, as shown in the flow chart presented inFig.1, the proposed AEFA was constructed to solve unconstraint optimization problems, whereas the OPP problem is a constraint optimization problem.

Based on the previous requirements, a modified AEFA is proposed in this paper to solve the OPP problem. The modified AEFA is constructed to modify the AEFA to be a binary AEFA and to enable AEFA to solve the constraint optimization problem such OPP problem. However, the flow chart of the modified AEFA is presented inFig.2. 


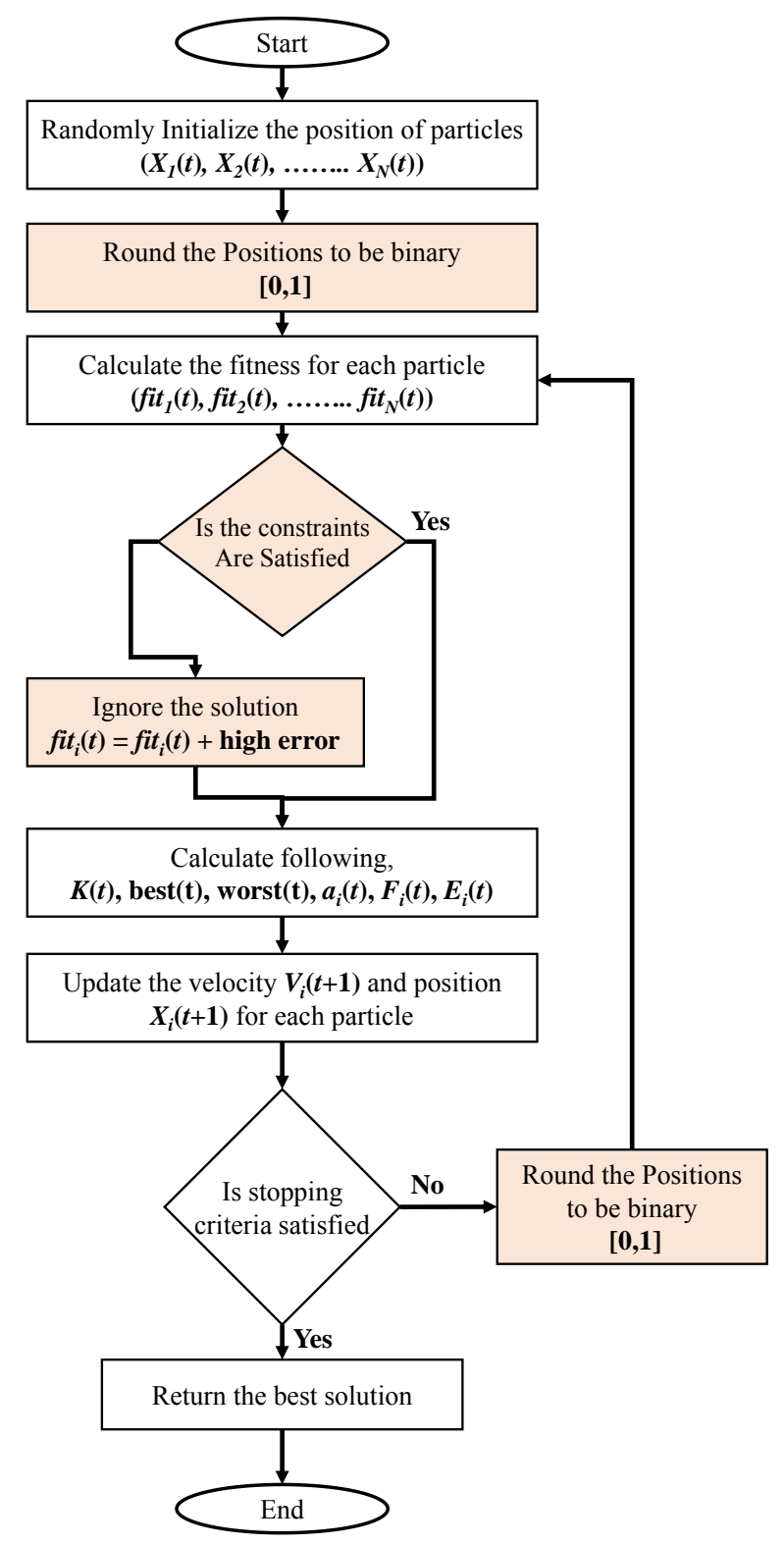

Fig. 2. Flow chart of modified AEFA to solve the OPP problem

IV.

\section{THE PROPOSED COST MODEL FOR PMU PLACEMENT}

The general formulation of the OPP problem founds the minimum number of PMUs as well as their locations in the entire system, where the objective function minimizes the number of PMUs as in Eq. (17), subjected to complete observability constraint as in Eq. (18),

$$
\min \sum_{i=1}^{n} x_{i}
$$

Subjected to,

$$
a_{i j} \cdot x_{i}^{T} \geq b_{i}^{T}
$$

Where, $a_{i j}$ is a binary connectivity matrix of the entire system which is defined as in Eq. (19), $x_{i}$ is a vector of length $\mathrm{n}$ indicates to PMU location as in Eq. (20), and $b_{i}$ is unit vector of length $n$.

$$
\begin{aligned}
& a_{i j}=\left\{\begin{array}{cc}
1 & \text { if bus } i=b u s j \\
1 & \text { if buses and are connected } \\
0 & \text { otherwise }
\end{array}\right. \\
& x_{i}=\left\{\begin{array}{cc}
1 & \text { if PMU is installed at bus } i \\
0 & \text { otherwise }
\end{array}\right.
\end{aligned}
$$

If all buses are observable, the observability constraint, in Eq. (18), will be equal or greater than 
one. Practically, the total cost of WAMS does not depends on the PMU cost only, but it depends on other important factors such as the availability of CT and VT, number of channels, communication infrastructure, and PDC cost. These additional parameters are changing from substation to others. In this paper, the total installation cost of WAMS is considered as objective function to determine the optimal number and location of PMUs in an electrical power system. The proposed cost model incorporates the PMU cost, PDC cost, and additional cost called upgrading cost. The proposed presented as:

$$
\text { Cost }=\sum_{i=1}^{N}\left(C_{P M U} x_{i}+C_{u p g}(i)\right)+C_{P D C}
$$

Where, $C_{P M U}$ is the PMU unit cost, $C_{P D C}$ is the PDC cost, $C_{\text {upg }}(i)$ is the additional cost which is function of bus number. In this study the additional cost $C_{u p g}(i)$ is different from substation to others. The additional cost represents the needed to upgrade some devices such as VT, CT, additional communication devices needed to upgrade the communication infrastructure. The additional cost given by:

$$
C_{u p g}(i)=C_{u p g} P_{i}
$$

Where, $C_{u p g}$ is a cost factor and $P_{i}$ is the number of branches in substation $i$. In the proposed model the cost data for PMU, PDC, and additional cost are taken from U.S. department of energy's office of electricity delivery and energy reliability [23], which are presented in Table. 1.

Table 1. Realistic cost of PMU, PDC, and additional cost [23].

\begin{tabular}{ccrl}
\hline Item & PMU $(\$)$ & PDC $(\$)$ & Additional Cost $(\$ /$ branch) \\
\hline Cost & 40,000 & 8,000 & 12,000 \\
V. RESULTS AND DISCUSSION & $\begin{array}{l}\text { systems. The lines data of the test systems are } \\
\text { obtained from [24]. The test systems information } \\
\text { included number of lines, number of ZIB, and ZIB }\end{array}$ \\
In this section, AEFA is used to solve the & $\begin{array}{l}\text { location are presented in Table. 2. Various } \\
\text { OPP problem with different models proposed in the }\end{array}$ \\
$\begin{array}{l}\text { present work. IEEE test systems are used to parameters have been tested to tune the AEFA. } \\
\text { demonstrate the ability AEFA to solve small and }\end{array}$ & $\begin{array}{l}\text { However, the parameter setting of AEFA is } \\
\text { large power system. The test systems include IEEE }\end{array}$
\end{tabular}
14 -bus, 30-bus, 39-bus, 57-bus, and 118-bus

Table 2. Test system information.

\begin{tabular}{cccc}
\hline Test System & NO. of Lines & NO. of ZIBs & ZIB locations \\
\hline IEEE 14-Bus & 20 & 1 & 7 \\
\hline IEEE 30-bus & 41 & 6 & $1,2,5,6,9,22,25,27,28$ \\
\hline IEEE 39-bus & 46 & 12 & $4,11,13,14,17,19,22$ \\
\hline IEEE 57-bus & 80 & 15 & $5,9,11,21,22,24,26,34,36,37,39,40,45,46,48$ \\
\hline IEEE 118-bus & 186 & 10 &
\end{tabular}

Table.3. Parameters setting of AEFA.

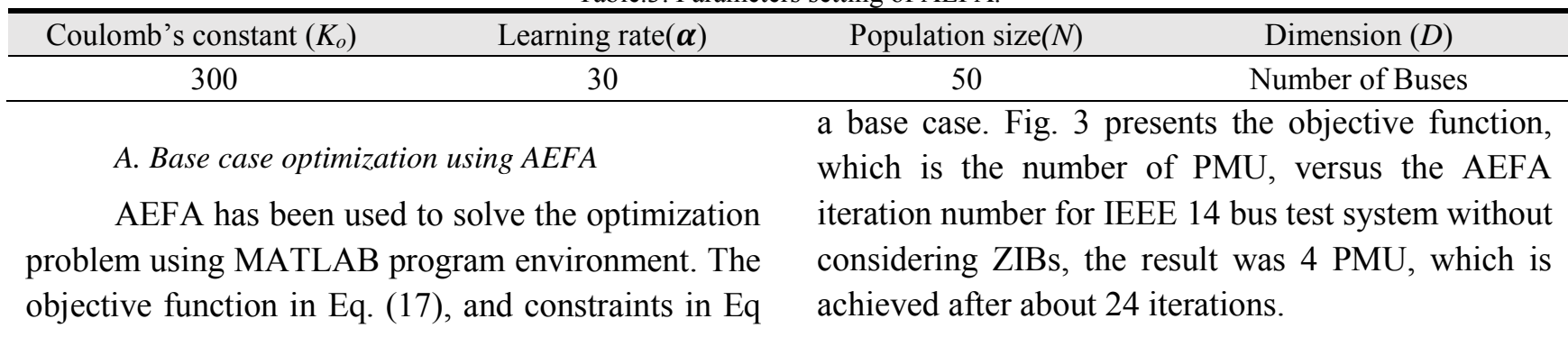
(18) were used to minimize the number of PMUs as 


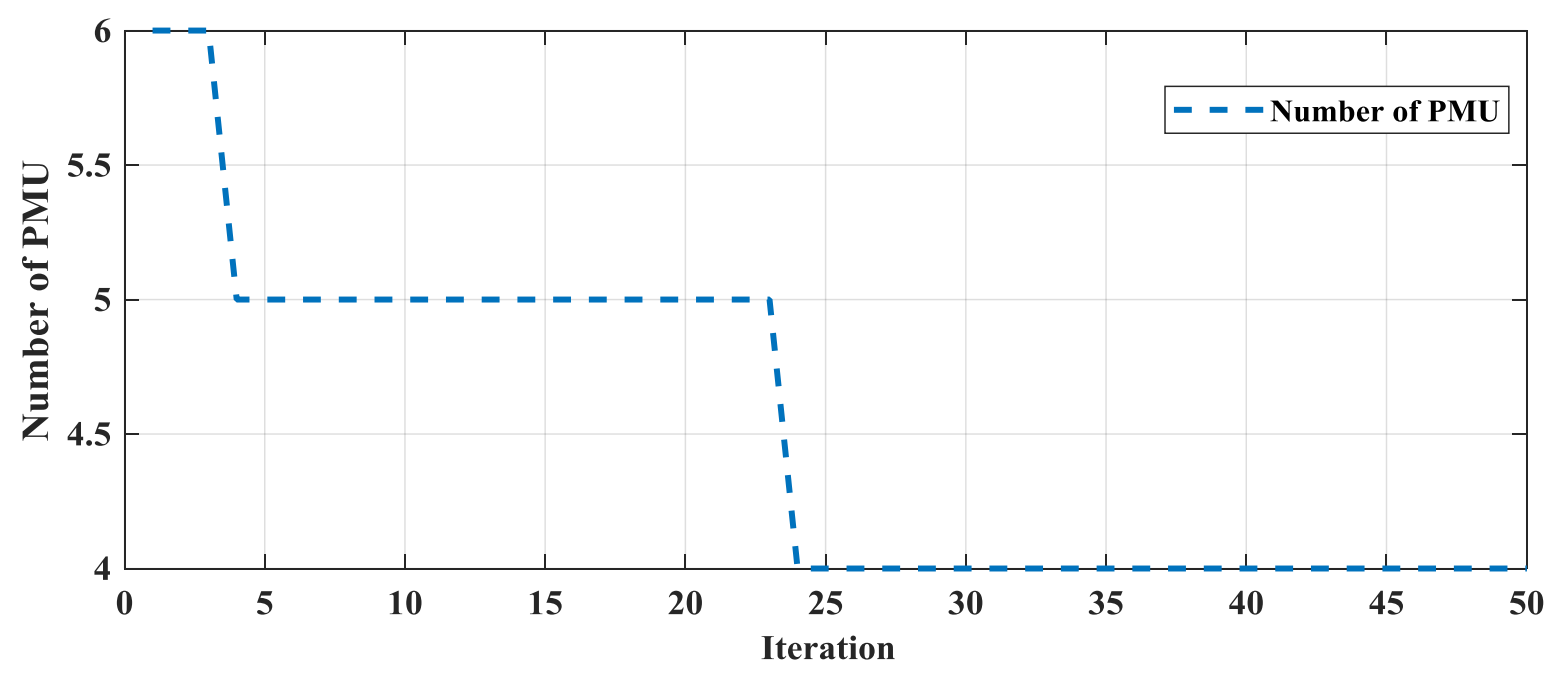

Fig. 3. Number of PMU using AEFA for IEEE 14 test system without ZIB considering.

Fig. 4 presents the number of PMUs versus the AEFA iteration number for IEEE 14 bus test system with considering ZIBs, where the result was 3 PMU, it was achieved after about 120 iterations. Fig. 5 presents the AEFA decreasing the number of PMU with iteration, it is observed that the number without
ZIB considering was 10 PMUs. On the other hand, the number of PMU with ZIB considering is 9 PMUs. However, the number of PMUs for 9, 14, 30, 39,57 , and 118 bus system are summarized in Table. 4.

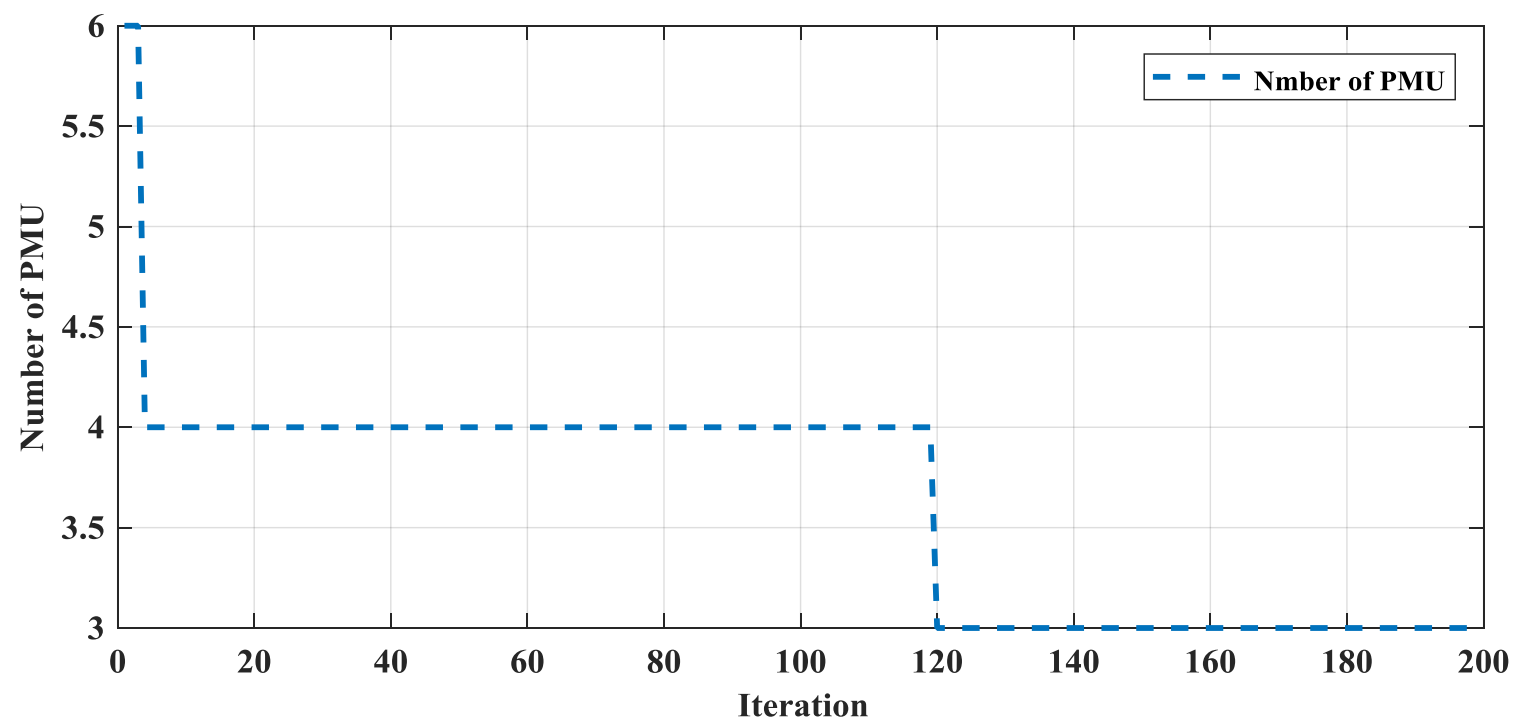

Fig. 4. Number of PMU using AEFA for IEEE 14 test system with ZIB. 


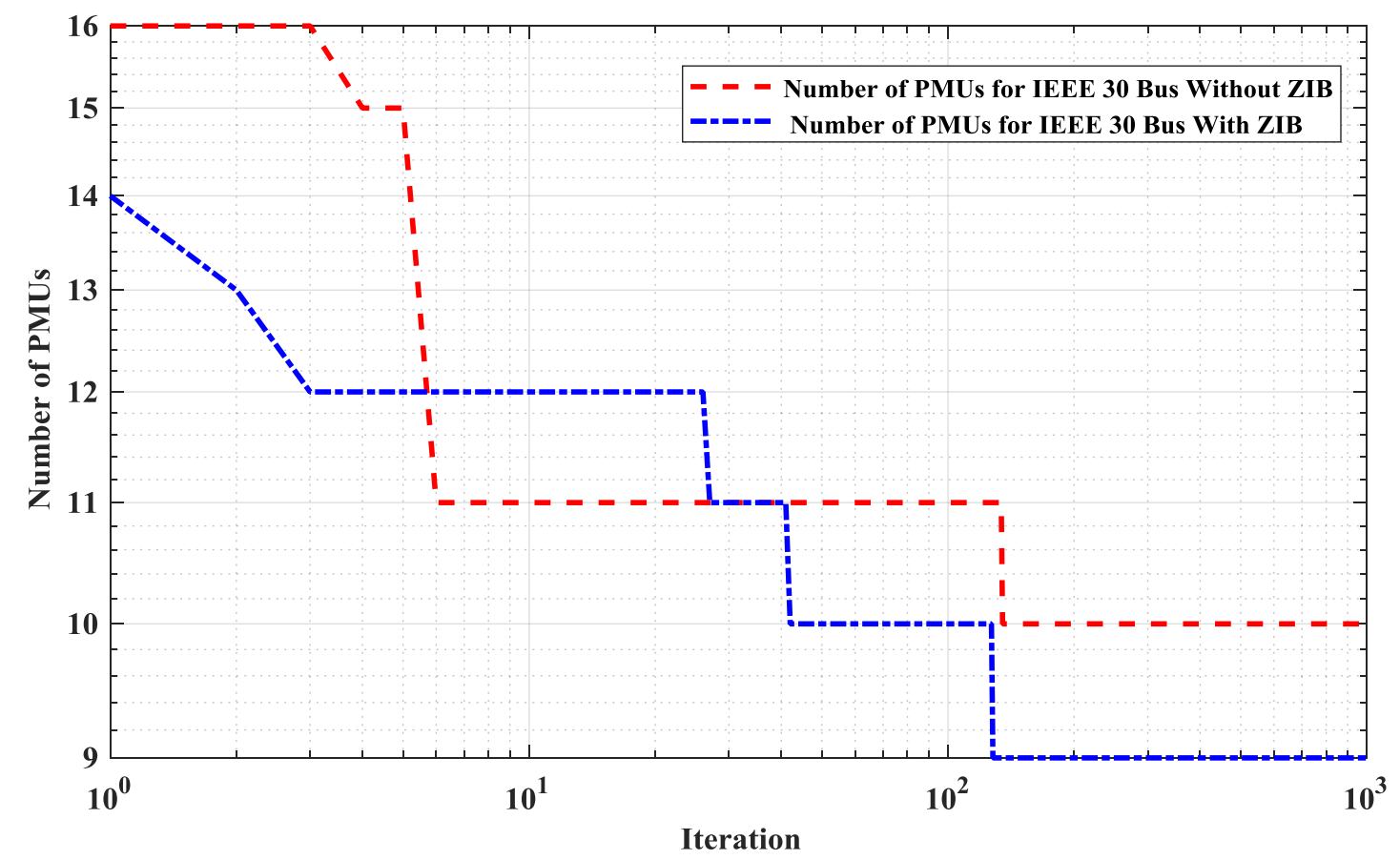

Fig. 5. Number of PMU using AEFA for IEEE 30 test system with and without considering ZIBs.

Table. 5 Presents a comparison between the AEFA and other optimization techniques such as integer linear programming ILP, genetic algorithm GA [17], and particle swarm optimization PSO [19]. It clearly shows that if the number of PMU is used to determine the optimal location of PMUs, all algorithm will obtain same number of PMUs.

'Table.4. PMUs placement for base case using AEFA

\begin{tabular}{|c|c|c|c|c|}
\hline \multirow{2}{*}{$\begin{array}{c}\text { Test } \\
\text { System }\end{array}$} & \multicolumn{2}{|r|}{ Without ZIBs } & \multicolumn{2}{|r|}{ With ZIBs } \\
\hline & $\begin{array}{c}\text { NO. } \\
\text { PMUs }\end{array}$ & PMUs Placement & $\begin{array}{l}\text { NO. } \\
\text { PMUs }\end{array}$ & PMUs Placement \\
\hline 9-Bus & 3 & $3,4,8$ & 2 & 4,7 \\
\hline 14-Bus & 4 & $2,6,7,9$ & 3 & $2,6,9$ \\
\hline 30-bus & 10 & $1,7,8,10,11,12,19,23,26,27$ & 7 & $2,4,10,12,18,24,27$ \\
\hline 39-bus & 13 & $\begin{array}{c}2,6,9,13,14,17,22,23,25,29,32, \\
33,34\end{array}$ & 9 & $3,8,11,13,15,20,23,24,29$ \\
\hline 57-bus & 17 & $\begin{array}{c}1,4,6,9,15,20,24,28,31,32,36 \\
38,41,47,51,53,57\end{array}$ & 13 & $\begin{array}{c}1,4,9,13,19,22,25,28,32,37,50,54 \\
56\end{array}$ \\
\hline 118-bus & 32 & $\begin{array}{c}3,8,11,12,17,21,27,31,32,34, \\
37,40,45,49,51,54,56,61,66,70 \\
71,75,77,80,83,86,89,92,96 \\
100,105,110\end{array}$ & 28 & $\begin{array}{c}3,8,11,12,17,21,27,31,32,34,37,40 \\
45,49,53,56,62,72,75,77,80,85,86 \\
90,94,102,105,110\end{array}$ \\
\hline
\end{tabular}

Table. 5. Comparison between the AEFA, ILP, GA, PSO results

\begin{tabular}{ccccc}
\hline TestSystem & ILP[6] & GA[17] & PSO [19] & AEFA \\
\hline 9-Bus & 3 & 3 & 3 & 3 \\
\hline 14-Bus & 4 & 4 & 4 & 4 \\
\hline 30-bus & 10 & 10 & 10 & 13 \\
\hline 39-bus & 13 & 13 & 13 & 17 \\
\hline 57-bus & 17 & 17 & 17 & \\
\hline
\end{tabular}




\section{8-bus}

B. Results for proposed cost model using AEFA

In this section the proposed cost model presented in Eq. (21) has solved using the modified AEFA. For more realistic the cost of PMUs and other related equipment are taken from U.S.
32 32 32

department of energy's office of electricity delivery and energy reliability [23], which are presented in Table. 1.The optimization results for IEEE 9, 14, 30, 39,57 , and 118 bus system have been presented in Table.

Table. 6. AEFA results for proposed cost model

\begin{tabular}{cccc}
\hline $\begin{array}{c}\text { Test } \\
\text { System }\end{array}$ & $\begin{array}{c}\text { Number of } \\
\text { PMU }\end{array}$ & $\begin{array}{c}\text { PMUs } \\
\text { Location }\end{array}$ & $\begin{array}{c}\text { Total } \\
\text { Cost }(\$)\end{array}$ \\
\hline 9-Bus & 3 & $3,4,8$ & 248,000 \\
\hline 14-Bus & 4 & $2,8,10,13$ & 336,000 \\
\hline 30-bus & 10 & $2,6,9,12,11,17,22,23,25,29,32,33,34$ & 876,000 \\
\hline 39-bus & 13 & $1,4,10,15,20,23,28,29,31,32,36,39,41,47,49,54$ & $1,080,000$ \\
\hline 57-bus & 17 & $1,5,9,11,12,17,21,23,28,30,34,37,42,45,49,53,56,62$, & $3,148,000$ \\
\hline 118 -bus & 32 & $64,68,71,75,77,80,85,87,91,94,101,105,110,115$ & $11,12,19,24,25,28,29$ \\
\hline
\end{tabular}

The minimum cost to achieve full observability for IEEE 9, 14, 30, 39, 57, 118 test systems were 0.248, $0.336,0.876,1.08,1.404,3.148$ million dollars, respectively. For more investigation, the results of PMUs placement that obtained by minimize the number of PMU have been used to calculate total cost of WAMS implementation in IEEE test systems. Here, the PMU, PDC, and additional cost are considered as in the proposed cost model. The calculated costs have been compared with total costs, which obtained by the modified AEFA. These comparisons are presented in Fig. 6.

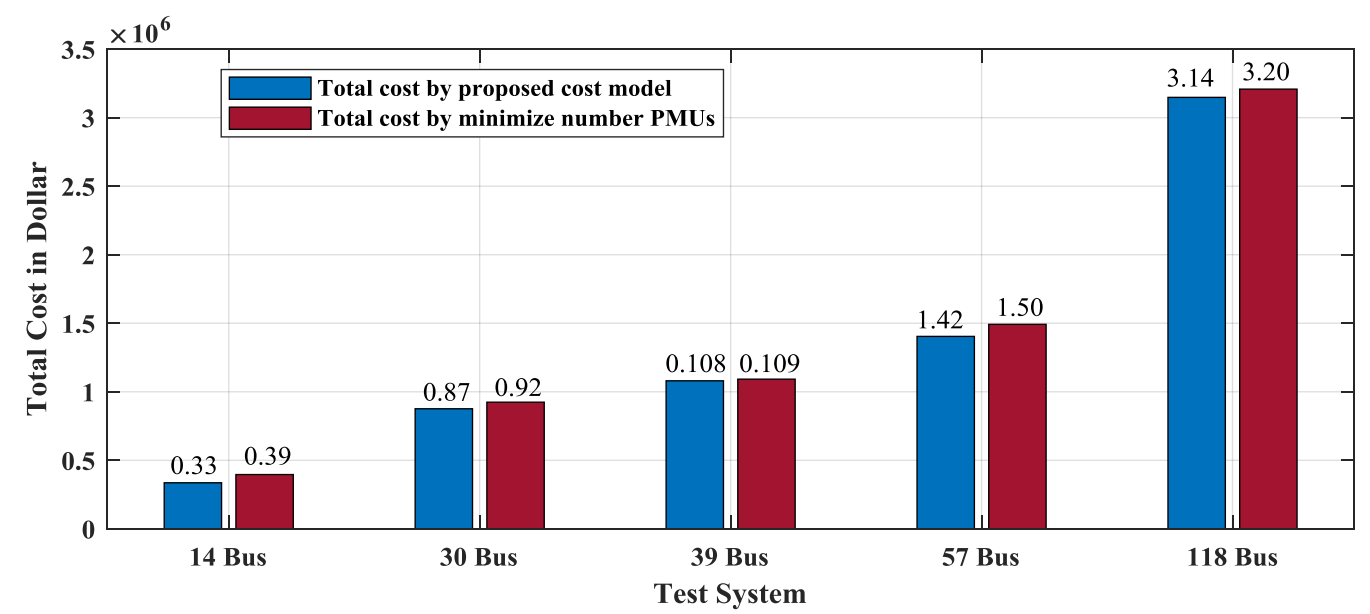

Fig. 6. Total cost of WAMS based on proposed cost model and conventional number of PMUs minimization.

Fig. 6 clearly shows that the proposed cost model enhances to find the optimal PMUs placement that decrease the total cost of WAMS implementation. On the other hand, the minimization of number of PMUs does not greatly influence on the cost compared with proposed cost model. For example, the proposed model decreased the total cost by $15 \%$ in IEEE 14 bus system.

\section{CONCLUSIONS}

In this paper, a realistic cost model was proposed to solve the OPP problem. The comprehensive cost model, which was proposed in 
this paper, aims to minimize the total cost of WAMS, where it includes the PMU, PDC, and other additionally costs. The proposed cost model has been solved using artificial electric field algorithm (AEFA), which is a novel optimization algorithm, it was proposed by 2019 .

In this paper a binary form of AEFA has been proposed to solve the general form OPP problem as well as the proposed one. The modified AEFA was implemented in MATLAB programming environment. The OPP results of modified AEFA have compared with other related solutions obtained with other optimization algorithms. As a result, the AEFA is efficient to solve the OPP problem. Additionally, the results of proposed OPP model demonstrate that the proposed model provides designers realistic overview about the total cost of WAMS implementation.

\section{REFERENCES}

[1] S. Chakrabarti and E. Kyriakides, "Optimal placement of phasor measurement units for power system observability," IEEE Transactions on Power Systems, vol. 23, no. 3, p. 1433-1440, 2008.

[2] L. Huang, Y. Sun, J. Xu, W. Gao, J. Zhang and Z. $\mathrm{Wu}$, "Optimal PMU placement considering controlled islanding of power system," IEEE Transactions on Power Systems, vol. 29, no. 2, p. 742-755, 2014.

[3] T. K. Maji and P. Acharjee, "Multiple solutions of optimal PMU placement using exponential binary PSO algorithm for smart grid applications," IEEE Trans. Ind. Appl, vol. 1, no. 99, p. 1-5, 2017.

[4] J. Aghaei, A. Baharvandi, A. Rabiee and M. A. Akbari, "Probabilistic PMU placement in electric power networks: An MILP-based multiobjective model," IEEE Trans. Ind. Informat, vol. 11, no. 1, pp. 332-341, 2015.

[5] E. Kyriakides and S. Chakrabarti, "'Optimal placement of phasor measurement units for power system observability," IEEE Transaction on Power System, vol. 23, no. 3, p. 1433-1440, 2008.

[6] F. Aminifar, A. Khodaei, M. Fotuhi-Firuzabad and a. M. Shahidehpour, "Contingency-constrained PMU placement in power networks," Transation on Power System, vol. 25, no. 1, p. 516-523, 2010.

[7] M. Hajian, A. M. Ranjbar, T. Amraee and a. B. Mozafari, "Optimal placement of PMUs to maintain network observability using a modified BPSO algorithm," International Journal of Electrical Power \& Energy System, vol. 33, no. 1, p. 28-34, 2011.
[8] M. B. Mohammadi, R. A. Hooshmand and F. H. Fesharaki, "A new approach for optimal placement of PMUs and their required communication infrastructure in order to minimize the cost of the wams," IEEE Trans. Smart Grid, vol. 7, no. 2, p. 8493, 2016.

[9] M. B. Mohammadi, R. A. Hooshmand and a. F. H. Fesharaki, "A new approach for optimal placement of PMUs and their required communication infrastructure in order to minimize the cost of the wams," IEEE Trans. Smart Grid, vol. 7, no. 2, p. 8493, 2016.

[10] Z. H. Rather, Z. Chen, P. Thgersen, P. Lund and a. B. Kirby, "Realistic approach for phasor measurement unit placement: Consideration of practical hidden costs," IEEE Trans. Power Del., vol. 30, no. 3, p. 3 $15,3-15$.

[11] B. Gou, "Generalized integer linear programming formulation for optimal PMU placement, vol. 23, no. 3, pp.," IEEE Transaction onPower System, vol. 23, no. 3, p. 1099-1104, 2008.

[12] B. Gou, "Optimal placement of PMUs by integer linear programming," IEEE Trans. Power System, vol. 23, no. 3, pp. 1525-1526, 2008.

[13] R. Kavasseri and S. K. Srinivasan, "Joint optimal placement of PMU and conventional measurements in power systems," in Proceedings of 2010 IEEE International Symposium on Circuits and Systems, Paris, 2010.

[14] S. Chakrabarti, D. Eliades, E. Kyriakides and M. Albu, "Measurement uncertainty considerations in optimal sensor deployment for state estimation," in Proc. IEEE Int. Symp. Intelligent Signal Processing, Alcala de Henares, 2007.

[15] P. Gopakumar, M. J. B. Reddy and D. K. Mohanta, "Novel multi-stage simulated annealing for optimal placement of PMUs in conjunction with conventional measurements," in 2013 12th International Conference on Environment and Electrical Engineering, Wroclaw, 2013.

[16] S. S. Geramian, H. A. Abyane and K. Mazlumi, "Determination of optimal PMU placement for fault location using genetic algorithm," in 2008 13th International Conference on Harmonics and Quality of Power, Wollongong, NSW, 2008.

[17] M. K. Paramathma and D. Devaraj, "Genetic Algorithm Based Approach for the Determination of Optimal Locations and Observability of Phasor Measurement Unit (PMU) Under Smart Grid Environment," in 2019 IEEE International Conference on Clean Energy and Energy Efficient Electronics Circuit for Sustainable Development (INCCES), Krishnankoil, India, 2019.

[18] S. Almasabi and a. J. Mitra, "A Fault-Tolerance Based Approach to Optimal PMU Placement," IEEE Transactions on Smart Grid, vol. 10, no. 6, pp. 60706079, 2019. 
[19] S. Kumari, P. Walde, A. Iqbal and A. Tyagi, "Optimal phasor measuring unit placement by binary particle swarm optimization," in 2017 8th International Conference on Computing, Communication and Networking Technologies (ICCCNT), Delhi, 2017.

[20] M. Dalali and H. Kazemi Karegar, "Optimal PMU placement for full observability of the power network with maximum redundancy using modified binary cuckoo optimisation algorithm," IET Generation, Transmission \& Distribution, vol. 10, no. 11, pp. 2817-2824, 2016.

[21] Anita and A. Yadav, "AEFA: Artificial electric field algorithm for global optimization," Swarm and Evolutionary Computation, vol. 48, no. 7, pp. 93$108,2019$.
[22] D. Holliday, R. Resnick and J. Walker, Fundamentals of physics, John Wiley and Sons, 1993.

[23] "Factors affecting PMU installation costs," U. S. Department of Energy, Office of Electricity Delivery and Energy Reliability, 2016.

[24] R. D. Christie, "Power Systems Test Case Archive," 1999. [Online]. Available: https://lamarr.ece.uw.edu/research/pstca/index.html.

\section{Creative Commons Attribution License 4.0 (Attribution 4.0 International, CC BY 4.0)}

This article is published under the terms of the Creative Commons Attribution License 4.0

https://creativecommons.org/licenses/by/4.0/deed.en_US 